European Association for the Development of Renewable Energies, Environment and Power Quality

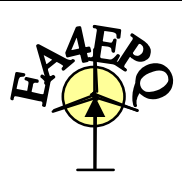

International Conference on Renewable Energies and Power Quality (ICREPQ'09)

Valencia (Spain), 15th to 17th April, 2009

\title{
Development of Switchable Transformer Research at University of South Australia
}

\author{
H. A. Dharmawan ${ }^{1}$ and S. A. M. Ali ${ }^{2}$ \\ ${ }^{1}$ School of Electrical and Information Engineering \\ University of South Australia, Adelaide, South Australia 5095 \\ Phone/Fax number: +61-8-83023346/+61-8-83023384, e-mail: dhaha001@students.unisa.edu.au \\ ${ }^{2}$ School of Electrical and Information Engineering \\ University of South Australia, Adelaide, South Australia 5095 \\ Phone/Fax number: +61-8-83023346/+61-8-83023384, e-mail: Sam.Ali@unisa.edu.au
}

\begin{abstract}
This paper describes research development focusing on the designs of switchable transformers and their control system at University of South Australia. Research development of switchable transformer has become one of the major research interests in school of Electrical and Information Engineering, University of South Australia since year 2000. Research in this area has been carried out through undergraduate to post graduate studies. Several transformer designs, starting from the rating of $1 \mathrm{kVA}$ to $10 \mathrm{kVA}$, have been introduced. Testing results from the designs have shown the improvement of the transformer efficiency during low or high loads. As the switchable transformers utilize several switching devices that need to be synchronously controlled, research on the development of the switchable transformer control systems has also been carried out. Now, in this school, a study of utilizing controller area network (CAN) as media to integrate modules in the control system is done. The study also focused on the remote monitoring and control system of the switchable transformer through a local area network (LAN).
\end{abstract}

\section{Key words}

Switchable transformer, Energy efficient, Research development.

\section{Introduction}

Switchable transformers are newly introduced types of transformer featuring several winding configurations that can be altered based on the variation of the loads[1]. Unlike windings in normal or conventional transformers which have fixed number of turns in their primary and secondary, winding configurations in switchable transformers provide higher number of turns for low loads and lower number of turns for high loads. Through these configuration implementations, amount of losses can be kept less than that of fixed number of windings. In other words, switchable transformers have higher efficiency than conventional transformers have. A design strategy to increase the number of turns in low loads is to provide extra turns of windings and this is referred to as series configurations. On the other hand, to lower the number of turn in high loads, some portions of turns of windings are configured in parallel and this is referred to as parallel configurations.

Figure 1 and 2 provide an example of how efficiency of a conventional transformer improved through utilization of a series winding configuration below 0.5 p.u. and parallel winding configuration above 0.6 p.u.[2]. The point of load where the winding configuration should be switched from series to parallel or vice versa is called switching point of switchable transformers. In this example the switching point is between 0.5-0.6 p.u. These graphs were visualized from losses measurement results through open and short circuit tests of a single-phase $1 \mathrm{kVA}$ switchable distribution transformer built in 2000 .

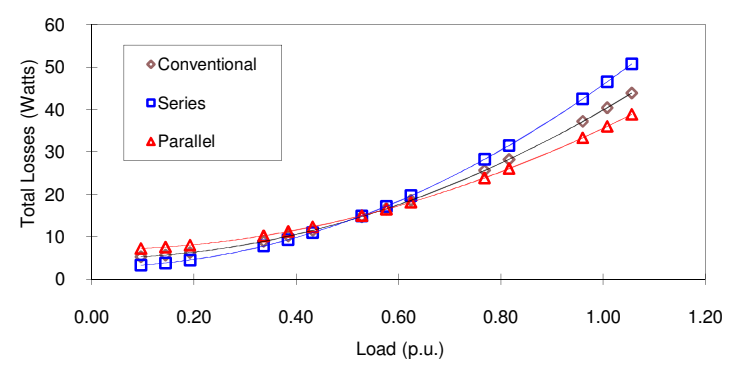

Fig. 1. Comparison of total losses of $1 \mathrm{kVA}$ conventional and switchable transformer[2]. 


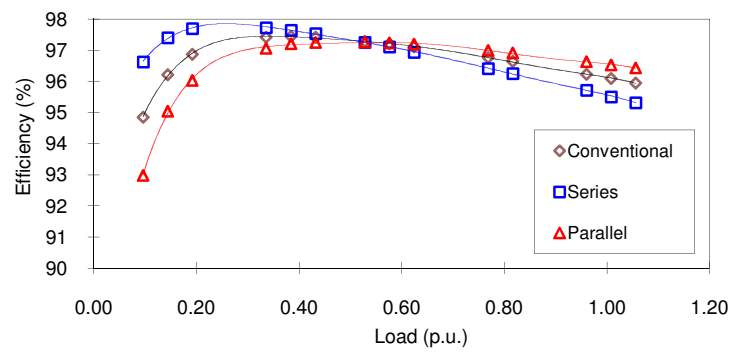

Fig. 2. Comparison of efficiency of $1 \mathrm{kVA}$ conventional and switchable transformer[2].

\section{Initial Studies of Switchable Transformer}

In 2000, through postgraduate study, a small single phase $1 \mathrm{kVA}$ switchable distribution transformer was designed and built in the school, with primary and secondary voltages of $415 \mathrm{~V}$ and $240 \mathrm{~V}$ respectively, using a uni-core shell-type configuration[2]. In the design process, techniques and calculations used were not far different with those for conventional transformers. The essential differences were the use of lower flux density at low loads and lower winding resistances at high loads through certain arrangement of tappings of windings in the switchable transformer. As can be seen from Fig. 3, the primary and secondary windings were made up of several tappings of the transformer which were connected through three switching devices (S1, S2 and S3) for both windings.
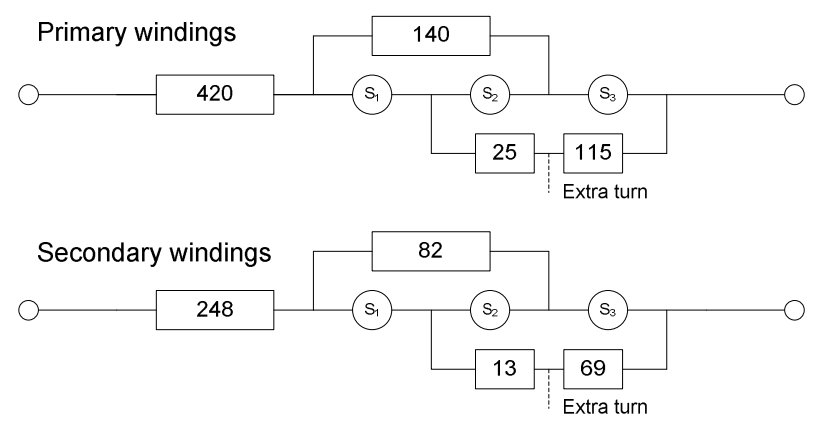

Fig. 3. Tappings of the $1 \mathrm{kVA}$ switchable transformer[2].

(Three tappings for each primary and secondary windings: $420,140,25+115,248,82,13+69)$

Table 1 summaries the characteristics of the transformer for the different configurations. It can be seen from this table the use of lower flux density (1.43T) for series configuration with the consequence of increasing the winding resistances. When this configuration is applied at low loads, the lower flux density provides lower core losses. As the core losses are dominant in low loads, the total losses will be less, although the winding resistances increase and cause more copper losses. On the other hand, the use of lower number of turns through parallel configuration will reduce copper losses which are dominant at high loads. Again, this will reduce total losses of the transformer at high loads. Using data from measurement results of open and short circuit tests, the losses and efficiency of the transformer configured in conventional, series and parallel are visualized in Figure 1 and 2.

Figure 4 shows the $1 \mathrm{kVA}$ transformer that was built and tested in 2000. A view of application software made and used in the design process of this transformer can be seen from Figure 5. This software reduced complication tasks in designing the transformer that included calculations and modifications. The design was in fact not straightforward and needed to perform some simulations to determine the best result. This application was however intended for only designs of switchable transformers having power rating up to $5 \mathrm{kVA}$ [2]. Through this application, users can enter specifications such as power rating, input \& output voltage, flux density, core type etc. in the specification section (see Figure 5 on the left column). After running the calculation, the program will show the summary of data as the results (see Figure 5 on right column). Users can scroll the window of the result in order to view the next parameters in this column. Setting for winding configurations, i.e conventional, series and parallel, is entered through the options section. Furthermore, percentage of additional turns in series configurations and percentage of turns which will be configured in parallel can also be set in this section. In designing the $1 \mathrm{kVA}$ switchable transformer, the best configuration of tappings for series and parallel configurations was mainly chosen based on the efficiency performances that could be seen through the efficiency figures which were generated automatically after running the calculation.

As additional design guide for further design of switchable transformers, the following limitations are mentioned in reference [2]:

1. Extra turns in series configuration windings will take more space compared to conventional windings. The design should be taken care of so that there will be sufficient window space left after installing whole turns.

2. As the lower number of turns in parallel configuration causes an increase in flux density, the design should makes sure that the flux density is still below its saturation region.

Table 1. Characteristic of the $1 \mathrm{kVA}$ transformer[2].

\begin{tabular}{|l|c|c|c|c|c|c|c|c|}
\hline \multirow{2}{*}{ Configuration } & \multicolumn{2}{|c|}{$\begin{array}{c}\text { Number } \\
\text { of Turns }\end{array}$} & \multirow{2}{*}{$\begin{array}{c}\text { Flux } \\
\text { Density } \\
(\mathrm{T})\end{array}$} & \multicolumn{2}{|c|}{$\begin{array}{c}\text { Measured } \\
\text { Winding } \\
\text { Res. }(\Omega)\end{array}$} & \multicolumn{3}{|c|}{$\begin{array}{c}\text { Switching } \\
\text { Devices } \\
\text { Status }\end{array}$} \\
\cline { 2 - 7 } & Pri. & Sec. & & Pri. & Sec. & S1 & S2 & S3 \\
\hline Series & 700 & 412 & 1.43 & 2.44 & 1.44 & off & on & off \\
\hline Conventional & 585 & 343 & 1.72 & 1.97 & 1.13 & off & on & off \\
\hline Parallel & 560 & 330 & 1.80 & 1.70 & 1.01 & on & off & on \\
\hline
\end{tabular}

Pri.: Primary, Sec.: Secondary. 

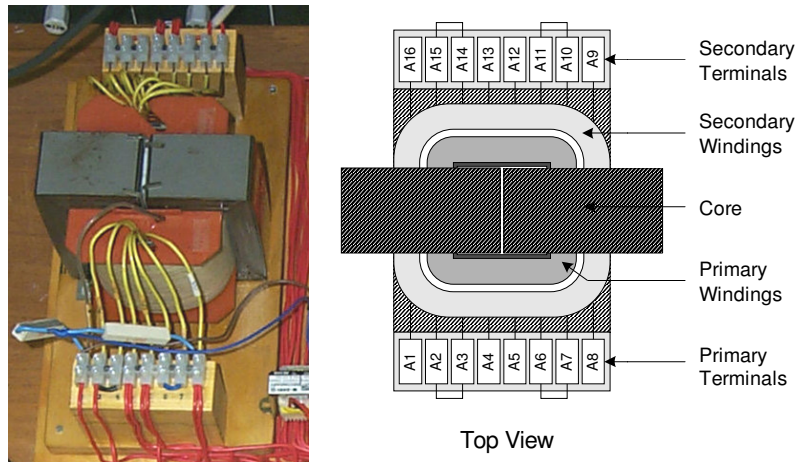

Top View

Fig. 4. The $1 \mathrm{kVA}$ transformer built and tested in 1999[2].

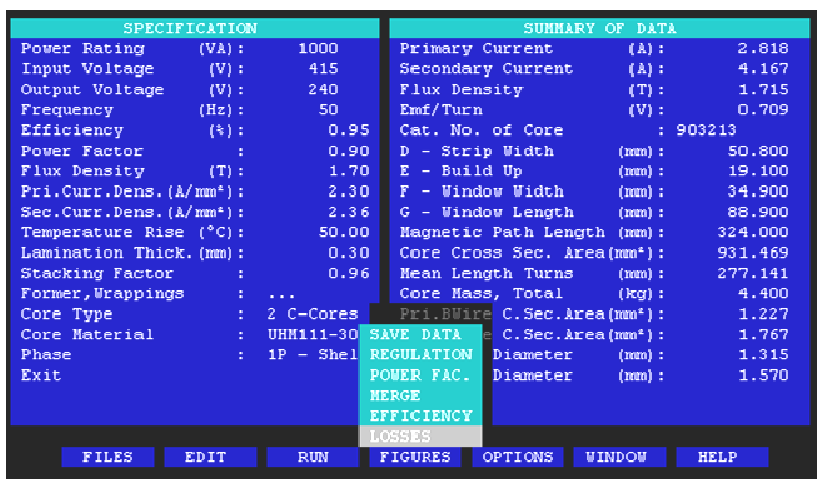

Fig. 5. Switchable transformer design software[2].

The design of the switchable transformer was continued up to year 2001, where a three-phase switchable transformer was constructed using three single-phase switchable transformers. Figure 6 shows the connection of this transformer and its measurement setup. In the experiment, a three-phase power supply and variable load were connected to the transformer. While applying variable loads, current and voltage signals were recorded through DAQ interface in a personal computer for analysis.

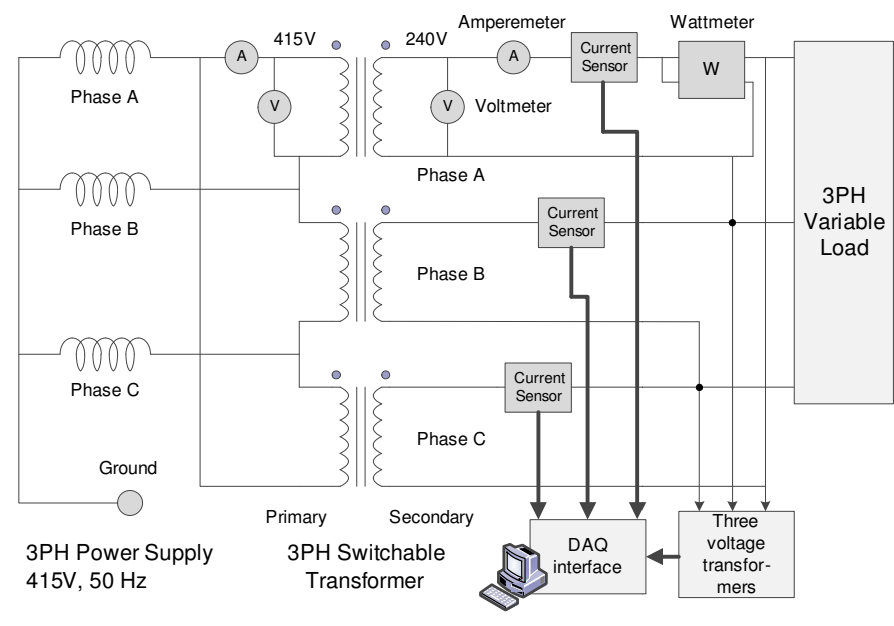

Fig. 6. Measurement setup of the three-phase switchable transformer.
Moreover in this year, a microcontroller is provided to control the switching devices in order to achieve smooth switching process of the switching devices[3]. Primary and secondary voltages as well as currents were sensed and passed through ADC to the microcontroller. The microcontroller provided triggers to the intended switching devices, based on the configuration that should be used. Eighteen SSRs (see Figure 7) were used for the switching devices and controlled synchronously by the microcontroller. As presented in [3], the smooth switching process from series to parallel configuration or vice versa could be made possible through the utilization of the microcontroller.

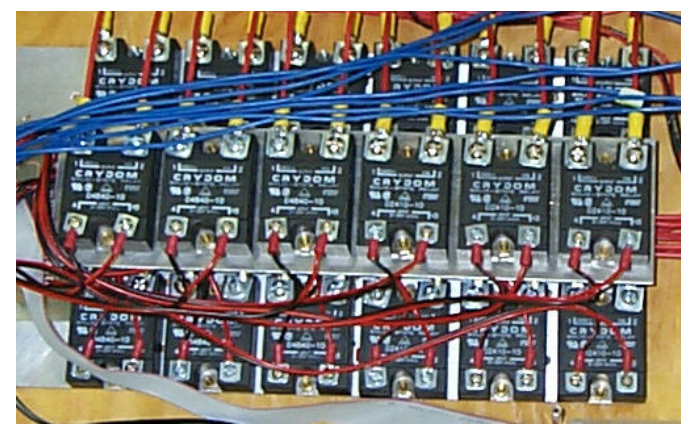

Fig. 7. The switching devices for the three-phase switchable transformer used in 2000.

\section{Further Development of Switchable Transformer}

Since year 2000 then, switchable transformers become major research interests in school of Electrical and Information Engineering, University of South Australia, and in 2002, a three-phase $3 \mathrm{kVA}$ switchable distribution transformer was designed and built. Photo of this transformer can be seen from Figure 8a. During this time, research on optimizing the design of the switchable transformer was also done and focused on modeling the magnetic flux in the transformer using Finite Element Analysis (FEA) software, i.e. Quickfield from Tera Analysis Ltd.[4]. By using this software, magnetic flux density in transformer's cores can be visualized at any moment in time. Research on optimization of transformer's cores was carried out by simulating the effects of winding configurations, performing geometrical adjustments and comparing the results visualized by the software.

As the research on switchable transformers continued, in 2003 a three-phase $10 \mathrm{kVA}$ switchable transformer was also built; see Figure 8b. The design was optimized through the use of the FEA software. Figure 9 shows tappings used for the $3 \mathrm{kVA}$ and $10 \mathrm{kVA}$ switchable transformers which basically had the same manner of configurations as the previous $1 \mathrm{kVA}$ switchable transformer. Specifications of these transformers are presented in Table 2 and 3. Testing results from these transformers also showed that higher 
efficiency performance can be achieved through their series and parallel configurations.

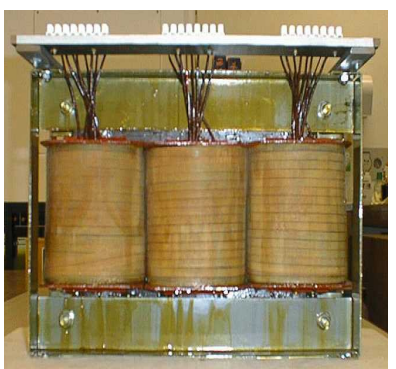

(a)

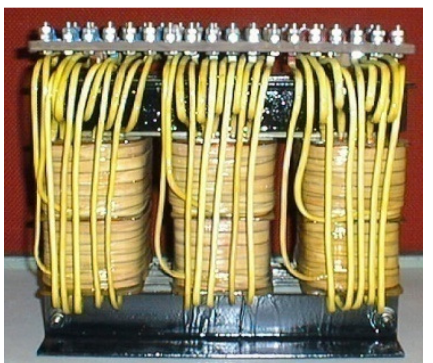

(b)
Fig. 8. The three-phase switchable transformers: (a) $3 \mathrm{kVA}$ built in 2002 (b) $10 \mathrm{kVA}$ built in 2003 .
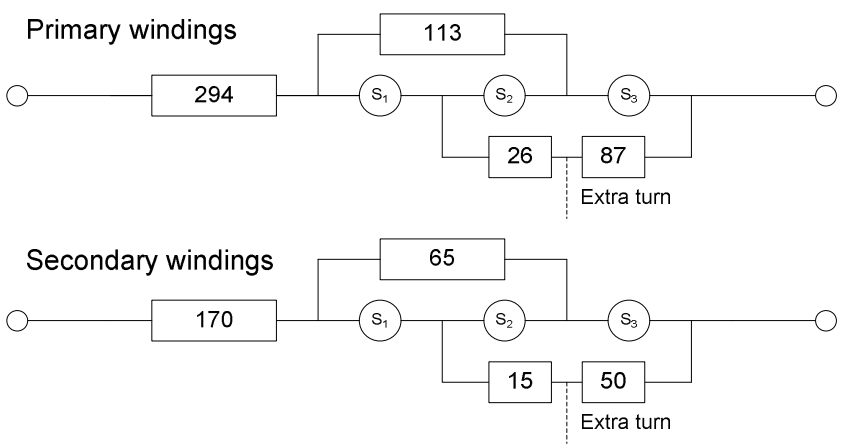

(a)
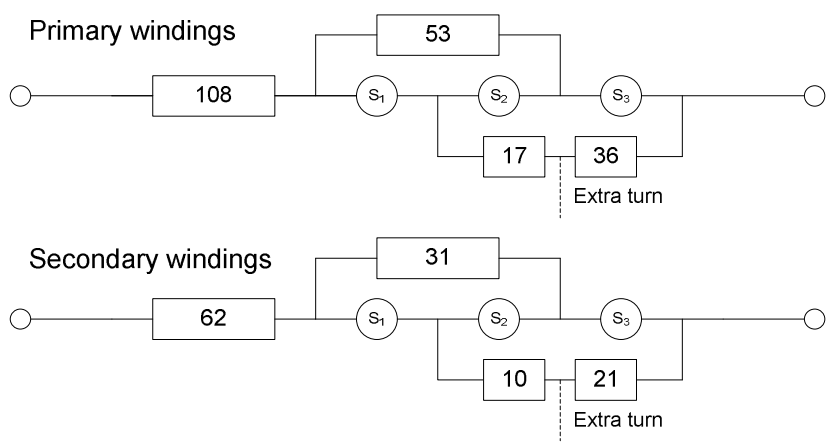

(b)

Fig. 9. The tappings of windings of the three-phase switchable transformers: (a) $3 \mathrm{kVA}$ built in 2002 (b) $10 \mathrm{kVA}$ built in 2003 .

Table 2. Characteristic of the $3 \mathrm{kVA}$ transformer[7].

\begin{tabular}{|l|c|c|c|c|c|}
\hline \multirow{2}{*}{ Config. } & \multicolumn{2}{|c|}{$\begin{array}{c}\text { Number } \\
\text { of Turns }\end{array}$} & \multirow{2}{*}{$\begin{array}{c}\text { Flux } \\
\text { Density }\end{array}$} & \multicolumn{2}{|c|}{$\begin{array}{c}\text { Measured } \\
\text { Winding Res. }(\Omega)\end{array}$} \\
\cline { 2 - 3 } \cline { 5 - 6 } & Pri. & Sec. & $(\mathrm{T})$ & Pri. Ph1,2,3 & Sec. Ph1,2,3 \\
\hline Series & 520 & 300 & 1.28 & $1.43,1.44,1.44$ & $0.60,0.60,0.60$ \\
\hline Conv. & 433 & 250 & 1.55 & $1.15,1.15,1.16$ & $0.48,0.49,0.49$ \\
\hline Parallel & 407 & 235 & 1.64 & $0.92,0.93,0.93$ & $0.39,0.39,0.39$ \\
\hline
\end{tabular}

Pri. Ph1,2,3: Primary Phase 1, 2 \& 3.

Sec. Ph1,2,3: Secondary Phase $1,2 \& 3$.
Table 3. Characteristic of the $10 \mathrm{kVA}$ transformer[4].

\begin{tabular}{|l|c|c|c|c|c|}
\hline \multirow{2}{*}{ Config. } & \multicolumn{2}{|c|}{$\begin{array}{c}\text { Number } \\
\text { of Turns }\end{array}$} & \multirow{2}{*}{$\begin{array}{c}\text { Flux } \\
\text { Density }\end{array}$} & \multicolumn{2}{|c|}{$\begin{array}{c}\text { Measured } \\
\text { Winding Res. }(\Omega)\end{array}$} \\
\cline { 2 - 3 } \cline { 5 - 6 } & Pri. & Sec. & $(\mathrm{T})$ & Pri. Ph1,2,3 & Sec. Ph1,2,3 \\
\hline Series & 214 & 124 & 1.17 & $0.30,0.30,0.30$ & $0.10,0.09,0.09$ \\
\hline Conv. & 178 & 103 & 1.41 & Not Available & Not Available \\
\hline Parallel & 161 & 93 & 1.55 & $0.18,0.18,0.18$ & $0.06,0.05,0.05$ \\
\hline
\end{tabular}

Pri. Ph1,2,3: Primary Phase 1, 2 \& 3.

Sec. Ph1,2,3: Secondary Phase 1, $2 \& 3$.

Not long after the 10kVA switchable transformer was built, some studies (including postgraduate and undergraduate studies) on development of a data acquisition for analyzing performances of the transformer were conducted. One of the studies utilizes a 16-bit PIC microcontroller as embedded processor. Besides controlling the transformer, through its built-in digital signal processing (DSP) engine, the microcontroller performed FFT calculations to retrieve harmonic contents in the primary and secondary voltages and current signals. This microcontroller then sent acquired and processed data to a PC through an RS232 connection. A DAQ hardware and LabView from National Instrument were combined to provide comparison in monitoring the performances of the transformer.

Recently, in 2005, a study on the implementation of an adaptive fuzzy logic controller using executable heterogeneous model was conducted. This model was intended to integrate the environmental, loading and electrical parameters of the switchable transformers[5][6]. Through the use of fuzzy logic controller, more optimized control could be expected. As the research continues, more complications arise in the control system such as the requirement for fast calculations, better user interface and communication. These complicated tasks are difficult to perform by using one microcontroller as implemented in the previous control systems. More reliable designs with reduced wirings are also expected.

A new idea is then proposed through post graduate study and used as further development of the control system. The new idea here is to utilize multi-processor and controller area network (CAN) in the system. These processors are programmed to perform fast acquisition, calculations, send triggers and synchronize on/off operations of switching devices, provide a comprehensive and easy user interface and provide a reliable way of communicating to a server. The controller area network is chosen as media to integrate the processors which are separated through some modules in the control system. CAN is considered to be used because it is widely used in industries, low-cost (by using only two wires) and provides reliable serial transmission through its sophisticated error detection and robust error handling mechanisms. Furthermore, CAN also immunes to electromagnetic interference. 
Figure 10 shows the current control system of switchable transformer. The embedded controller is divided into several modules: high current controller, data acquisition (DAQ) for phase $1,2 \& 3$ and user interface \& RTU. The high current controller provides synchronized triggers to the 18 switching devices that connect the tappings of windings of the transformer. These switching devices are turned on/off based on the information that is directly sent by the three DAQ modules through CAN bus. Data processing, such as FFT calculation, is carried out in the DAQ modules. Beside transmitting the acquired parameters and processed data (temperature, voltages and currents) to the high current controller module and to the user interface \& RTU module, the DAQ modules provide exchange information between them self. The user interface module \& RTU module provides easy user interface to operators and connection to a server. The server can also get direct access to all the modules through CAN bus. Some client and server applications are developed to enable remote monitoring and control through the local area network (LAN) in the school.

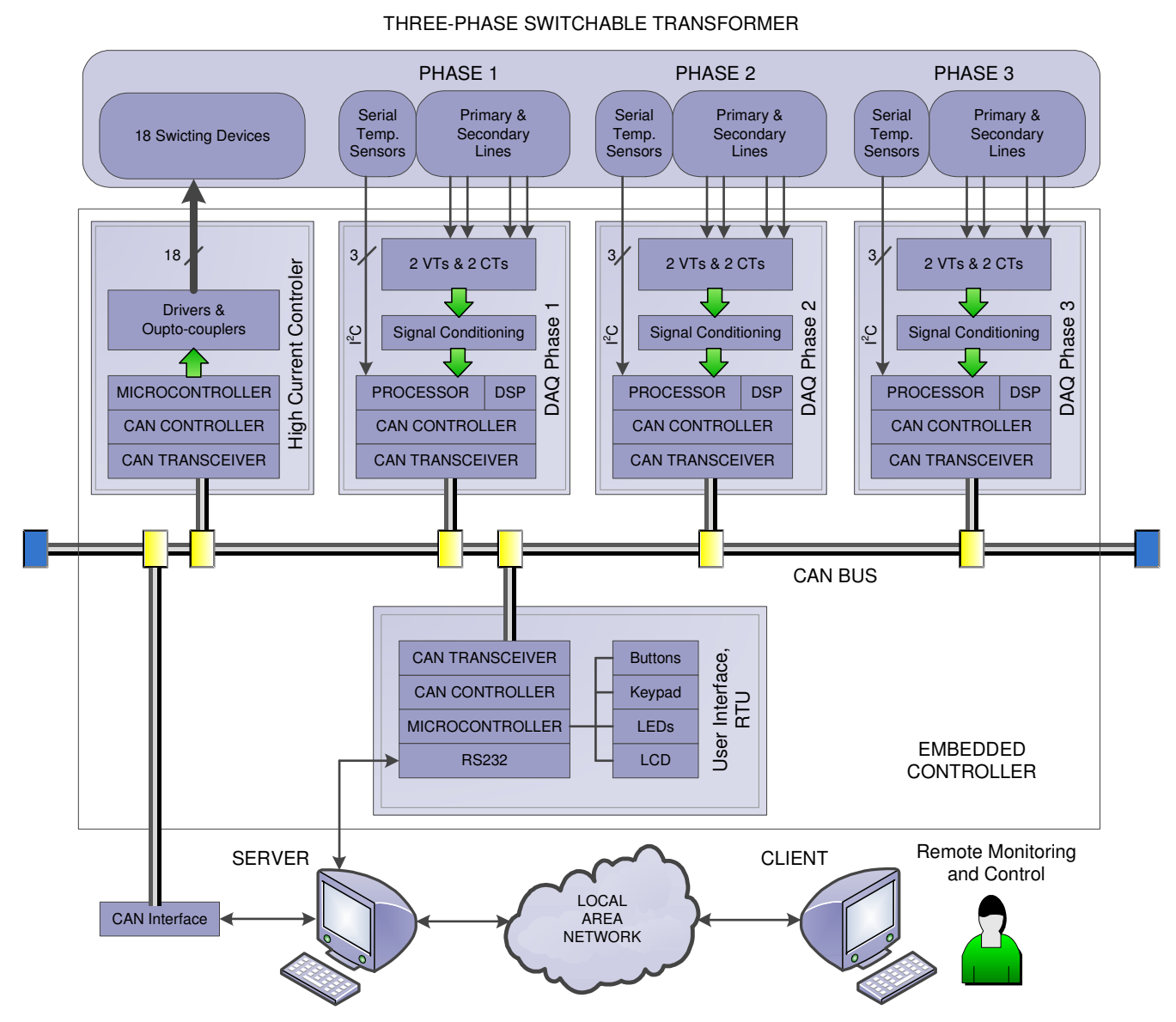

Fig. 10. Current control system of switchable transformer

\section{Conclusion}

Switchable transformers have become one of major research interests in school of Electrical and Information Engineering, University of South Australia since year 2000. Attempts have been made to provide better designs with higher power rating of switchable transformers and enhance reliability of the control system of the switchable transformers. Testing results have shown that the switchable transformers provide better characteristic in terms of efficiency performances during low or high loads.
This promises acceptance of using the switchable transformers in industries.

\section{Refferences}

[1] Ali, S.A.M. \& Dharmawan, H. A., "Energy Efficient Switchable Transformer", Applied Electromagnetics and Mechanics: Proceedings of the 10th International Symposium on Applied Electromagnetics and Mechanics, 2001, pp.29-30, Japan (T Takagi, M Uesaka, Eds).

[2] Dharmawan, H. A., "Micro-controlled Energy Efficient Switchable Transformer", Masters Thesis, University of South Australia, 2001. 
[3] Dharmawan, H. A. \& Ali, S.A.M., "Design of Switchable Transformer Control System Employing the Microcontroller 80C552", International Journal of Applied Electromagnetics and Mechanics, Japan, Vol. 13 (2002) pp. 99-106 ISBN 1586033271.

[4] Kelly, G. B., "Flux Modelling and Optimisation of 3 Phase Transformers using Finite Element Analysis", Project Report, University of South Australia, 2003.

[5] Kiefer, R. H \& Ali, S.A.M. " Heterogeneous Modeling of an Intelligent Switchable Energy Efficient Distribution Transformer Inclusive of Environmental and Loading Conditions", Proceedings, ICIS 2005 (International conference on intelligent systems Kuala Lumpur, Malaysia 1-3 December 2005, pp 1-6.

[6] Kiefer, R. H \& Ali, S.A.M. "Executable Heterogeneous Model of an Adaptive Fuzzy Logic Controller and Switchable Energy Efficient Distribution Transformer", ICEM-06 (International conference on electrical machines), Crete Island Greece, 2-5 September 2006.

[7] Ali, S.A.M \& Smith, J.K.E "The Practical design and application of a three phase, $3 \mathrm{kVA}$, energy efficient distribution transformer", Journal of Applications of Electromagnetic Phenomena in Electrical and Mechanical Systems" JSAEM and Kanazawa University, Japan, 2003, pp. 307-314.
[8] Ali, S.A.M, \& Klaer D. "Design \& Implementation of a 3Phase Energy Efficient Switchable Transformer Controller Using the PIC Microcontroller 18F8720", 2004 International conference on power system technology, 2124 November 2004, Singapore, pp.1-6.

[9] Ali, S.A.M \& Pham, H., "Design and Implementation of Smart PIC Micro-controlled 3-Phase Energy Efficient Distribution Transformer", Proceedings of 15th Symposium on Electromagnetics and Dynamics, 28-30 May 2003, Kanazawa Japan, pp 327-330.

[10] Ali, S.A.M \& Pham, H., "Testing and Evaluation of a 3Phase, 3 kVA Energy Efficient Distribution Transformer", Proceedings of 15th Symposium on Electromagnetics and Dynamics,28-30 May 2003, Kanazawa, Japan, pp.323-325.

[11] Kiefer, R. H \& Ali, S.A.M. "Three-Phase Switchable Distribution Transformers: Economics, Plus a Fuzzy Logic Control and Condition Monitoring System", The Third IASTED International Conference on Power and Energy Systems, 3-5 September 2003, Marbella, Spain, pp.516-520. 